\title{
A legislação e a sistematização da assistência de enfermagem
}

Alba Lucia Bottura Leite de Barros' Juliana de Lima Lopes ${ }^{2}$

O processo de enfermagem é o método utilizado para sistematizar a assistência de enfermagem no Brasil. Dessa forma, é imprescindível que os profissionais de enfermagem conheçam e apliquem as normas regulamentadoras do exercício. A Resolução Cofen 358/2009 estabelece que o processo de enfermagem deve ser realizado de modo deliberado e sistemático em todos os ambientes públicos ou privados em que ocorre o cuidado profissional de enfermagem. Ainda destaca as cinco etapas: coleta de dados (ou histórico), diagnóstico, planejamento, implementação e avaliação.

Descritores: Enfermagem, Ética de Enfermagem, Legislação de Enfermagem.

\section{The legislation and the systematization of nursing care}

The nursing process is the method used to systematize the nursing care in Brazil. This way, it is necessary that the nursing professionals know and apply the rules of the actions. The resolution Cofen 358/2009 establishes that the nursing process must be carried out deliberately and systematic in all public or private environments where there is a nursing professional care. Yet, it emphasizes the five stages: nursing data collection (or history), nursing diagnosis, nursing planning, implementation and nursing evaluation.

Descriptors: Nursing, Nursing Ethic, Nursing Legislation.

\section{La legislación y la sistematización de la asisténcia de enfermería}

El proceso de enfermería es el método utilizado para sistematizar la asisténcia de enfermería en Brasil. Desta forma, es imprescindible que los profesionales de enfermería conoscan y apliquem las normas reglamentadoras del ejercício. La Resolución Cofen 358/2009 estableces que el Proceso de enfermería deba ser realizado de modo deliberado y sistemático en todos los ambientes públicos ou privados en que ocurre el cuidado profesional de enfermería. Aunque destaca las cinco etapas: colecta de datos, diagnóstico, planeamiento, implementación y evaluación de enfermería.

Descriptores: Enfermería, Ética de Enfermería, Legislación de Enfermería.

\section{INTRODUÇÃO}

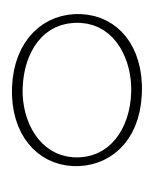
método utilizado para sistematizar a assistência de enfermagem é o Processo de Enfermagem (PE), forma de tomada de decisões que se apoia nos passos do método científico ${ }^{(1)}$. A Sistematização da Assistência de Enfermagem (SAE) vem ocorrendo desde Florence Nightingale, quando, ao participar como voluntária na Guerra da Crimeia com outras 38 mulheres, em 1854, conseguiu reduzir a mortalidade local de $40 \%$ para $2 \%(2)$. Florence preconizava que as enfermeiras deveriam estar submetidas a uma forte organização disciplinar ${ }^{(3)}$.

Atualmente, o PE vem sendo amplamente estudado e aplicado nos serviços de saúde no Brasil e no mundo. No Brasil, o modelo mais conhecido para a implantação do PE é o proposto por Horta ${ }^{(4)}$, que contém as seguintes fases: a) histórico de enfermagem, b) diagnóstico de enfermagem, c) plano assistencial, d) prescrição de enfermagem, e) evolução de enfermagem e f) prognóstico de enfermagem.

Entre as diversas vantagens da SAE destaca-se a elevação da qualidade da assistência de enfermagem, beneficiando tanto o paciente, por meio de um atendimento individualizado, quanto o enfermeiro, mostrando a importância do processo de enfermagem ${ }^{(1)}$.

A prática da assistência de enfermagem vai além do modelo biomédico. Ela é baseada e instrumentalizada por um referencial próprio, criado e construído pelos profissionais de enfermagem, que possibilita a união da teoria à prática. $\mathrm{O}$ uso de marcos conceituais explícitos na prática assistencial altera, também, a estrutura da forma da assistência, possibilitando ação participativa e crítica, embasada em conceitos científicos, exigindo maior conhecimento da disciplina de enfermagem ${ }^{(5)}$.

Além dessa habilidade técnica, é imprescindível que os profissionais de enfermagem conheçam e apliquem as normas regulamentadoras do exercício, dos direitos e das obrigações profissionais. Do ponto de vista ético, é esperado que o enfermeiro utilize sua criatividade ao gerenciar as ações assistenciais, ao tomar decisões e ao adequar os recursos humanos e materiais de que dispõe, assegurando um atendimento das necessidades dos pacientes com isenção de riscos quando esses forem previsíveis ${ }^{(6)}$.

Nesse contexto, o enfermeiro deve garantir a segurança e a integridade do paciente. De acordo com o Código de Ética de Profissionais de Enfermagem, as responsabilidades e os deveres desses profissionais, entre outros, são: "Assegurar uma assistência de enfermagem livre de danos decorrentes de imperícia, negligência ou imprudência" e "proteger o cliente contra danos decorrentes de imperícia, negligência

1 Professora titular da Escola Paulista de Enfermagem da Universidade Federal de São Paulo - Unifesp (SP), Brasil.

2 Enfermeira do Instituto do Coração - Incor. Doutoranda do programa de pós-graduação de enfermagem da Universidade Federal de São Paulo - Unifesp (SP), Brasil. 
ou imprudência por parte de qualquer membro da equipe de saúde" ${ }^{\prime \prime 7}$. As ações dos profissionais de enfermagem devem fundamentar-se nos valores da profissão e no Código de Ética, assegurando a promoção, proteção, recuperação e reabilitação das pessoas, respeitando os preceitos éticos e legais. Nesse sentido, a Lei do Exercício Profissional de Enfermagem (Lepe) no 7498/1986 estabelece as competências dos profissionais de enfermagem e a responsabilidade no agir com base nas competências técnicas, éticas, políticas ou relacionais de cada um. De acordo com essa lei, cabe ao enfermeiro o gerenciamento das ações de enfermagem ao planejar, executar, avaliar e discutir os resultados das condutas de enfermagem propostas com sua equipe. Ainda, o enfermeiro poderá delegar determinadas atribuições ao técnico ou ao auxiliar de enfermagem, sob sua supervisão, quando não forem ações privativas do enfermeiro ${ }^{(8)}$. O controle, a previsão e a atuação do enfermeiro nas ocorrências éticas envolvendo os profissionais de enfermagem perpassam as ações gerenciais desse profissional como líder da equipe de enfermagem e responsável pela supervisão e capacitação de recursos humanos em enfermagem. Assim, o enfermeiro torna-se responsável pelas ações de enfermagem, ao executá-las ou delegálas na equipe de enfermagem ${ }^{(6)}$.

A Lepe descreve as atividades privativas do enfermeiro como: direção do órgão de enfermagem integrante da estrutura básica da instituição de saúde, pública e privada, e chefia de serviço e de unidade de enfermagem; organização e direção dos serviços de enfermagem e de suas atividades técnicas e auxiliares nas empresas prestadoras desses serviços; planejamento, organização, coordenação, execução e avaliação dos serviços da assistência de enfermagem; consultoria, auditoria e emissão de parecer sobre matéria de enfermagem; consulta de enfermagem; prescrição da assistência de enfermagem; cuidados diretos de enfermagem a pacientes graves com risco de vida; cuidados de enfermagem de maior complexidade técnica e que exijam conhecimentos de base científica e capacidade de tomar decisões imediatas ${ }^{(8)}$.

Diante do exposto, este artigo tem como objetivo apresentar aspectos da Lepe e da SAE com a finalidade de instrumentalizar o enfermeiro no cotidiano de seu trabalho.

\section{A SAE e o Exercício Profissional}

Para que o enfermeiro possa tomar as decisões, tem de se basear em conhecimentos científicos, intensificando o pensamento crítico e o raciocínio clínico. Conhecimentos e procedimentos teoricamente organizados, sistematizadose sempre reformulados se constituem em base segura para a ação eficiente ${ }^{(9)}$.

A aplicação de uma assistência de enfermagem sistematizada é a única possibilidade de o enfermeiro atingir sua autonomia profissional e constitui a essência de sua prática profissional. Desde 1986, o planejamento da assistência é uma imposição legal: "O enfermeiro exerce todas as atividades de enfermagem, cabendo-lhe privativamente: o planejamento, organização, coordenação, execução e avaliação dos serviços da assistência de enfermagem"(8).

A Resolução Cofen no 272/2002 reforça a importância e a necessidade de planejar a assistência de enfermagem e dispõe que "a implementação da SAE deve ocorrer em toda instituição da saúde, pública e privada"(10) e que as ações privativas do enfermeiro são "a implantação, planejamento, organização, execução e avaliação do processo de enfermagem", que compreende a consulta de enfermagem (histórico, exame físico, diagnóstico de enfermagem, prescrição de enfermagem e evolução de enfermagem) e o relatório de enfermagem.

\section{Etapas do Processo de Enfermagem}

Em 2009, a Resolução Cofen no 358/2009 revogou a Resolução Cofen $n^{\circ} 272 / 2002^{(10)}$. De acordo com essa resolução, O PE "deve ser realizado, de modo deliberado e sistemático, em todos os ambientes, públicos ou privados, em que ocorre o cuidado profissional de enfermagem". E afirma que o PE se organiza em cinco etapas ${ }^{(11)}$ :

I - Coleta de Dados de Enfermagem (ou Histórico de Enfermagem): processo deliberado, sistemático e contínuo, realizado com o auxílio de métodos e técnicas variadas, que tem por finalidade a obtenção de informações sobre a pessoa, a família ou a coletividade humana e sobre suas respostas em um dado momento do processo de saúde e doença;

II - Diagnóstico de Enfermagem: processo de interpretação e agrupamento dos dados coletados na primeira etapa, que culmina com a tomada de decisão sobre os conceitos diagnósticos de enfermagem que representam, com mais exatidão, as respostas da pessoa, família ou coletividade humana em um dado momento do processo de saúde e doença, e que constitui a base para a seleção das ações ou intervenções com as quais se objetiva alcançar os resultados esperados;

III - Planejamento de Enfermagem: determinação dos resultados que se esperam alcançar e das ações ou intervenções de enfermagem que serão realizadas face às respostas da pessoa, família ou coletividade humana em um dado momento do processo de saúde e doença, identificadas na etapa de Diagnóstico de Enfermagem;

IV - Implementação: realização das ações ou intervenções determinadas na etapa de Planejamento de Enfermagem;

V-Avaliação de Enfermagem: processo deliberado, sistemático e contínuo de verificação de mudanças nas respostas da pessoa, família ou coletividade humana em um dado momento do processo de saúde e doença, para determinar se as ações ou intervenções de enfermagem alcançaram o resultado esperado, e de verificação da necessidade de mudanças ou adaptações.

Na Resolução Cofen n 272/2002, destaca-se a terceira geração do $P E$, com o enfoque nos resultados dos pacientes. Nela, são utilizadas as classificações de diagnósticos, intervenções e resultados. Além disso, o raciocínio clínico baseia-se na avaliação de um primeiro resultado, através de indicadores de resultados 
para um determinado diagnóstico, e seu progresso é julgado após a realização das intervenções de enfermagem ${ }^{(12)}$.

Ao enfermeiro incumbe a liderança na execução e avaliação do $P E$, de modo a alcançar os resultados esperados, cabendoIhe, privativamente, o diagnóstico de enfermagem acerca das respostas da pessoa, família ou coletividade humana em um dado momento do processo de saúde e doença, bem como a prescrição das ações ou intervenções de enfermagem a serem realizadas ${ }^{(11)}$.

Destaca-se nessa resolução(11), no artigo $3^{\circ}$ e $4^{\circ}$, que o PE "deve estar baseado num suporte teórico que oriente a coleta de dados, o estabelecimento de diagnósticos de enfermagem e o planejamento das ações ou intervenções; e que forneça a base para a avaliação dos resultados de enfermagem alcançados". Essa resolução enfatiza a necessidade do uso de sistemas de classificações de diagnósticos, intervenções e resultados como estrutura teórica, possibilitando a avaliação do cuidado de enfermagem e identificando, portanto, os fenômenos da prática assistencial.

\section{Considerações finais}

Frente ao exposto, pode-se afirmar que a SAE é considerada a metodologia de trabalho mais conhecida e aceita no mundo, facilitando a troca de informações entre enfermeiros de várias instituições e garantindo a qualidade da assistência, uma vez que esse processo nos permite diagnosticar as necessidades, fazer a prescrição adequada dos cuidados e avaliar a evolução do cliente. A aplicação do processo de enfermagem proporciona ao enfermeiro a possibilidade da prestação de cuidados individualizados, uma vez que suas ações são sistematizadas e inter-relacionadas, visando à assistência ao ser humano ${ }^{(13)}$.

Entretanto, apesar das diversas vantagens do $\mathrm{PE}$, ainda existem problemas encontrados na prática clínica, como número insuficiente de profissionais, falta de treinamentos institucionais, impresso inadequado, poucos recursos e ausência de padronização de linguagem nas instituições ${ }^{(14-}$ 16). Mesmo com o empenho do Conselho e de toda a classe profissional, percebe-se que se trata de um conhecimento que, apesar de ter sido introduzido na década de 1970, ainda apresenta uma enorme lacuna entre a produção do conhecimento e sua aplicabilidade na prática diária do enfermeiro(16).

No entanto, é necessário que os enfermeiros envolvidos com sua aplicação se comprometam em articular suas práticas com a filosofia institucional, repensando o processo de trabalho em saúde ${ }^{(17)}$.

\section{Referências}

1. Cunha SMB, Barros ALBL. Análise da implementação da sistematização da assistência de enfermagem, segundo o Modelo Conceitual de Horta. Rev Bras Enferm. 2005;58(5):568-72.

2. Lira N, Bomfim MES. História da enfermagem e legislação. Rio de Janeiro: Cultura Médica; 1989.

3. Lunardi Filho WD. O mito da subalternidade do trabalho da enfermagem à medicina. $2^{\mathrm{a}}$ ed. Pelotas: Universitária; 2004.

4. Horta WA. Processo de Enfermagem. São Paulo (SP): EPU; 1979.

5. Paim L, Trentini M. Indo além do modelo teórico - uma experiência de

ligação teórico-prática na assistência de enfermagem. Texto \& Contexto Enferm. 1993;2(1):13-32.

6. Freitas GF, Oguisso T. Ocorrências éticas com profissionais de

enfermagem: um estudo quantitativo. Rev Esc Enferm USP. 2008;42(1):34-40. 7. Conselho Federal de Enfermagem. Resolução Cofen n² 240/2000. Código de Ética dos Profissionais de Enfermagem [Internet]. [citado em 2010 Jul 14]. Disponível em: http://www.portalcofen.gov.br/Site/2007/print. asp?articlelD=7069.

8. Conselho Federal de Enfermagem. Lei Cofen nº 7.498/86.

Regulamentação do exercício de enfermagem [Internet]. [citado em 2010 Jul 14]. Disponível em: http://www.portalcofen.gov.br/Site/2007/materias. asp?ArticleID=22\&section ID=35.

9. Souza MF. Teorias de enfermagem importância para a profissão. Acta Paul Enferm. 1988;1(3):63-5.

10. Conselho Federal de Enfermagem. Resolução Cofen n` 272/2002. Sistematização da Assistência de Enfermagem - SAE - nas Instituições de Saúde Brasileiras [Internet]. [citado em 2010 Jul 14]. Disponível em: http://
www.portalcofen.gov.br/Site/2007/materias.asp?ArticlelD=7100\&sectionID $=34$.

11. Conselho Federal de Enfermagem. Resolução Cofen n 358/2009. Sistematização da Assistência de Enfermagem e a implementação do Processo de Enfermagem em ambientes, públicos ou privados, em que ocorre o cuidado profissional de Enfermagem [Internet]. [citado em $2010 \mathrm{Ju}$ 14]. Disponivel em: http://www.portalcofen.gov.br/Site/2007/materias.asp? ArticlelD=10113\&sectionID=34

12. Barros ALBL. Classificações de diagnóstico e intervenção de enfermagem: Nanda-NIC. Acta Paul Enferm. 2009;22(Esp. 70 anos):864-7. 13. Andrade JS, Vieira MJ. Prática assistencial de enfermagem: problemas, perspectivas e necessidade de sistematização. Rev Bras Enf. 2005;58(3):261-5.

14. Barros ALBL. O trabalho docente assistencial de enfermagem no hospital São Paulo da Unifesp/EPM [tese]. São Paulo: Universidade Federal de São Paulo, Escola Paulista de Medicina; 1998.

15. Takahashi AA, Barros ALBL, Michel JLM, Souza MF. Dificuldades e facilidades apontadas por enfermeiras de um hospital de ensino na execução do processo de enfermagem. Acta Paul Enferm. 2008;21(1):32-8. 16. Figueiredo RM, Zem-Mascarenhas SH, Napoleão $A A$, Camargo $A B$. Caracterização da produção do conhecimento sobre sistematização da assistência de enfermagem no Brasil. Rev Esc Enferm USP. 2006;40(2):299-303.

17. Koerich MS, Backes DS, Nascimento KC, Erdmann AL. Sistematização da assistência: aproximando o saber acadêmico, o saber-fazer e o legislar em saúde. Acta Paul Enferm. 2007;20(4):446-51. 